\title{
Real Time PCR; A new, fast and accurate technique in diagnosing the severe type of hemophilia A patients
}

\author{
Sayed Hamid Mosavi ${ }^{1 *}$, Seyed Alireza Mesbah-Namin ${ }^{2}$ \\ 1- Medical Research Center, Kateb University, Kabul, Afghanistan \\ 2- Department of Clinical Biochemistry, Faculty of Medical Sciences, Tarbiat \\ Modares University, Tehran, Iran
}

\begin{abstract}
Introduction: Hemophilia A (HA) is one of the most deleterious X-linked bleeding disorders in male patients. Early and rapid detection could provide management strategies for this disease. This study aims to improve the classic method for detection of important intron 22 inversion mutations (INV22).

Materials and Methods: Whole blood samples were taken from 21 male children with a history of severe HA, referred to the Iranian comprehensive hemophilia care center. Two groups were involved for detection of INV22 and two methods were examined in a double-blind manner. The first method used a classical method, inverse PCR, and the second method was Real-time inverse PCR.

Results: Results showed that both methods could separately detect the INV22 in 11 out of 21 patients with severe HA (52\%) in the same accuracy, but with this difference that only one day was needed for detecting of INV22 by Real-time inverse PCR and on other hand 3 days were needed for the classical methods.

Concusion: Because of the medical importance of early detection or prenatal/postnatal diagnosis of this disease, this study recommends the Real-time inverse PCR technique for the rapid detection of this mutation in well-equipped genetic laboratories everywhere.

Key words: Hemophilia A, Factor VIII, Intron 22 Inversion, Real-time inverse PCR
\end{abstract}

\footnotetext{
*-dr.mousavi@kateb.edu.af (Corresponding Author)
} 


\section{Introduction}

Hemophilia A (HA) is the most common bleeding disorders in developing countries caused by a deficiency/defect in coagulation factor VIII (FVIII) (1). The frequency of this disease is about 1 in 5,000-10,000 newborns. Spontaneous bleeding episodes after minor trauma was shown in patients with severe HA. (2). The patients have to undergo the substitution coagulation factor for their lifelong, through injections of FVIII into the bloodstream $(3,4)$. For diagnosing the disease, measurement of the activated prothrombin thromboplastin time (aPTT) comes first, which could be prolonged and also prothrombin time (PT), which is generally normal. Then, the estimation of FVIII activity is important, which will reduce this disease (5). The severity of the disease is determined, in terms of the level of normal FVIII activity in plasma, severe having $<1 \%$ of normal levels, moderate $1-5 \%$, and mild 6-30\% (6). The FVIII gene located at Xq28 position at the telomeric end of the $\mathrm{X}$ chromosome comprising 26 exons and 25 introns encoding 2332 amino acids. There are high GC regions within the 9.1 $\mathrm{kb}$ coding region of FVIII make it hyper mutable representing 140 different potential base-pair changes (7). The known and causative mutations of the FVIII gene in severe HA are very heterogeneous (8), but the most prevalent HA mutation is the intron 22 inversion (Inv22), which accounts for about 42-50\% of severe cases worldwide (9) and $39.5 \%$ in Iran (10). Intron 22 inversion originates in male germ cells (11) and from homologous recombination between the int22h-1 region within the FVIII locus and either int22h-2 (Inv22 type 2) or int22h-3 (Inv22 type 1), which lie approximately $400 \mathrm{~kb}$ distal to FVIII (12). The classical method for detecting of INV22 mutation in patients with hemophilia is the inverse PCR (I-PCR), but, some researchers have tried to modify this method using other techniques such as longdistance PCR (LD-PCR) (13) or LD Real-time PCR (14), which are relatively rapid, but their inappropriate sensitivities may cause 
difficulty in the interpretation of results. This report presents a reliable method for detecting Inv22, using Real-time inverse-PCR in individuals affected with severe HA.

\section{Materials and methods}

Whole blood samples of 21 unrelated male babies have randomly taken over one year (started in 2015) with their parents' consent. They were affected by severe HA, and referred to the Iranian Comprehensive Haemophilia Care Center (ICHCC).

Some of the samples were analyzed immediately, while others stored at minus $80^{\circ} \mathrm{C}$. Genomic DNA extracted from the samples using the High Pure PCR Template-Preparation Kit (Roche Applied Science, Germany) according to the manufacturer's instructions. The quality and quantity of extracted DNA assessed by agarose gel electrophoresis and spectrophotometrically, and stored in aliquots at $-20 \circ \mathrm{C}$ until required. The study is carried out on two independent groups to detect the known inversion mutation in intron 22 (INV22) in a double-blind manner using two method. The first method was detecting classic method, I-PCR, and the second method was detecting Real-time inverse PCR. For the first group I-PCR method was used that is described by Rossetti et al with some modification (15). Without purification step of digestion of DNA by a restriction enzyme, the $B c l I$-digested DNA ligated with T4 DNA ligase and B-rings amplified using special primers under standard PCR condition. The sequences of the primers used in this method are as follows: IU (intragenic upstream; 5 -CCTTTCAACTCCATCTCCAT3; Accession No. BX842559, nucleotides 35744 -35763); ID (intragenic downstream; 5 -ACATACGGTTTAGTCACAAGT-3; Accession No. BX842559; nucleotides 14622-14602), and ED (extragenic downstream; 5 -TCCAGTCACTTAGGCTCAG-3 , proximal Accession No. BX682237, nucleotides 15364 -15347; distal accession No. BX276110, nucleotides $14604-14621)$. As mentioned in 
the procedure, it is expected that the products with $487 \mathrm{bp}$ band specified for the normal and 559-bp for the mutant alleles visualized in $1.5 \%$ agarose gel electrophoresis. Other group used the Real-time PCR after getting B-ring for detecting INV22 in affected samples with severe HA ( named Real-time inverse PCR method). They had to have more purified DNA samples. Therefore, $2 \mu \mathrm{g}$ of extracted DNA digested overnight in a $50 \mu \mathrm{l}$ reaction volume containing $B c l$ I enzyme $(10 \mathrm{U} / \mu \mathrm{l}$; Fermentas ) under conditions recommended by the manufacturer. Then, fragmented DNA was undergone to the ligation reaction by T4 DNA ligase ( Fermentas ), and incubated overnight at $4{ }^{\circ} \mathrm{C}$. Ligated DNA extracted in phenol-chloroform. Real time-inverse PCR performed on the 3-6 $\mu$ l of eluted DNA (nearly $200 \mathrm{ng}$ of B-rings) in a $20 \mu \mathrm{l}$ of reaction volume, using specific primers as previously described and using Light Cycler ${ }^{\circledR}$ Fast Start DNA Master plus SYBR Green. The initial denaturation step was $10 \mathrm{~min}$ at $95^{\circ} \mathrm{C}$ followed by 40 cycles of $95{ }^{\circ} \mathrm{C}$ for $20 \mathrm{~s}, 65^{\circ} \mathrm{C}$ for $20 \mathrm{~s}$, and $72{ }^{\circ} \mathrm{C}$ for $20 \mathrm{~s}$; After that, melting curve obtained for the validity of the amplification results. Finally, $5 \mu 1$ of Real-time inverse PCR products were visualized on the $1.5 \%$ agarose gel electrophoresis.

\section{Results}

The results of this study considered in two concepts: First, the rate of prevalence of the INV22 mutation in patients with severe HA. Second, evaluation of using the recommended method for rapid detection of INV22 mutation in the same patients. The results show that both classical and recommended method accurately detected the INV22 mutation in patients with severe HA. In this regard, only 11 out of 21 (52\%) patients had the INV22 mutation in both experimental groups. Consequently, using Real-time inverse PCR on all of the samples, we obtained the same results in the less-time consuming manner (for only one day instead of 3 days) in comparison with I-PCR method. Figures 
$1 \mathrm{a}$ and $1 \mathrm{~b}$ show the bands for samples with (559 bp) and without INV22 mutation (487 bp) on the gel agarose electrophoresis, respectively. Figure 2 shows the melting curves of most of the samples applied in Real-time inverse PCR with $\left(\mathrm{Tm}=83^{\circ} \mathrm{C}\right)$ and without $\left(\mathrm{Tm}=85^{\circ} \mathrm{C}\right)$ INV22 mutation.

\section{Discussion}

We have described a reliable method (Real-time inverse PCR) for genotyping of INV22 mutation in patients with severe HA. Results of the first step of this study showed that 11 out of 21 DNA samples (52\%) had the Inv22 mutation in subjects affected with severe HA which were confirmed by I-PCR method and Real-time inverse PCR. Regarding the frequency of INV22 mutation, results indicate that the prevalence of INV22 mutation is variable, ranging from $36 \%$ in Spain patients to $55 \%$ in the Netherlands (16). Our results in this study are similar to those of Xue F. et al in China (2010), which was 51\% (17). The advantage of described method is the rapid and much more sensitive diagnosis of Inv22 mutation in comparison with I-PCR. Meanwhile, the time required for I-PCR exceeds 3 days including overnight incubation to achieve BclI digestion, the same for fragments ligation, two-step purification, doing PCR and finally post PCR phases, while Real-time inverse PCR amplification usually takes only one day. This is the first report showing that the I-PCR method can be improved by using Realtime inverse PCR

\section{Conclusion}

This study recommends the Real-time inverse PCR technique for the rapid detection of mutation in well-equipped genetic laboratories.

\section{Conflict of Interest}

The authors declared no conflict of interest. 


\section{Acknowledgment}

We cordially thank Professor Fereydoun Ala from the Iranian Comprehensive Hemophilia Care Center (ICHCC) and his colleagues for their help in sample collection, processing, and storage of the samples and providing all facilities required for this study.

\section{References}

1. Pezeshkpoor B, Pavlova A, Oldenburg J, El-Maarri O. F8 genetic analysis strategies when standard approaches fail. Hamostaseologie. 2014;34(2):167-73.

2. Keeney S, Mitchell M, Goodeve A. The molecular analysis of haemophilia A: a guideline from the UK haemophilia centre doctors' organization haemophilia genetics laboratory network. Haemophilia : the official journal of the World Federation of Hemophilia. 2005;11(4):38797.

3. Hedner U, Ginsburg D, Lusher JM, High KA. Congenital Hemorrhagic Disorders: New Insights into the Pathophysiology and Treatment of Hemophilia. Hematology Am Soc Hematol Educ Program. 2000:241-65.

4. Ng HJ, Lee LH. Haemophilia in 21st century Singapore. Ann Acad Med Singapore. 2009;38(4):378-2.

5. Franchini M, Targher G, Montagnana M, Lippi G. Laboratory, clinical and therapeutic aspects of acquired hemophilia A. Clin Chim Acta. 2008;395(1-2):14-8.

6. Pruthi RK. Hemophilia: a practical approach to genetic testing. Mayo Clin Proc. 2005;80(11):1485-99.

7. Human Gene Mutation Database website 2013 [ 25th Feb]. Available from: http://www.hgmd.org.

8. Pinto P, Ghosh K, Shetty S. F8 gene mutation profile in Indian hemophilia A patients: Identification of 23 novel mutations and factor VIII inhibitor risk association. Mutation research. 2016;786:27-33.

9. Lakich D, Kazazian HH, Jr., Antonarakis SE, Gitschier J. Inversions disrupting the factor VIII gene are a common cause of severe haemophilia A. Nat Genet. 1993;5(3):236-41. 
10. 1Rastegar Lari G, Enayat MS, Arjang Z, Lavergne JM, Ala F. Identification of intron 1 and 22 inversion mutations in the factor VIII gene of 124 Iranian families with severe haemophilia A. Haemophilia :the official journal of the World Federation of Hemophilia. 2004;10(4):4101.

11. Rossiter JP, Young M, Kimberland ML, Hutter P, Ketterling RP, Gitschier $\mathrm{J}$, et al. Factor VIII gene inversions causing severe hemophilia A originate almost exclusively in male germ cells. Human molecular genetics. 1994;3(7):1035-9.

12. Naylor JA, Buck D, Green P, Williamson H, Bentley D, Giannelli F. Investigation of the factor VIII intron 22 repeated region (int22h) and the associated inversion junctions. Human molecular genetics. 1995;4(7):1217-24.

13. Liu Q, Nozari G, Sommer SS. Single-tube polymerase chain reaction for rapid diagnosis of the inversion hotspot of mutation in hemophilia A. Blood. 1998;92(4):1458-9.

14. Vidal F, Sanchez-Garcia JF, Farssac E, Ramirez L, Gallardo D. Rapid single-step detection of the inversion hotspot of mutation in hemophilia A by real-time PCR. Journal of thrombosis and haemostasis : JTH. 2005;3(12):2822-3.

15. Rossetti LC, Radic CP, Larripa IB, De Brasi CD. Genotyping the hemophilia inversion hotspot by use of inverse PCR. Clin Chem. 2005;51(7):1154-8.

16. Gouw SC, van den Berg HM, Oldenburg J, Astermark J, de Groot PG, Margaglione M, et al. F8 gene mutation type and inhibitor development in patients with severe hemophilia A: systematic review and metaanalysis. Blood. 2012;119(12):2922-34.

17. Xue F, Zhang L, Sui T, Ge J, Gu D, Du W, et al. Factor VIII gene mutations profile in 148 Chinese hemophilia A subjects. European journal of haematology. 2010;85(3):264-72. 
Figure 1. Gel agarose electrophoresis for patient samples with INV22 (a) and without INV22 (b) after doing Real-time inverse PCR. M= DNA 50 bp ladder marker.

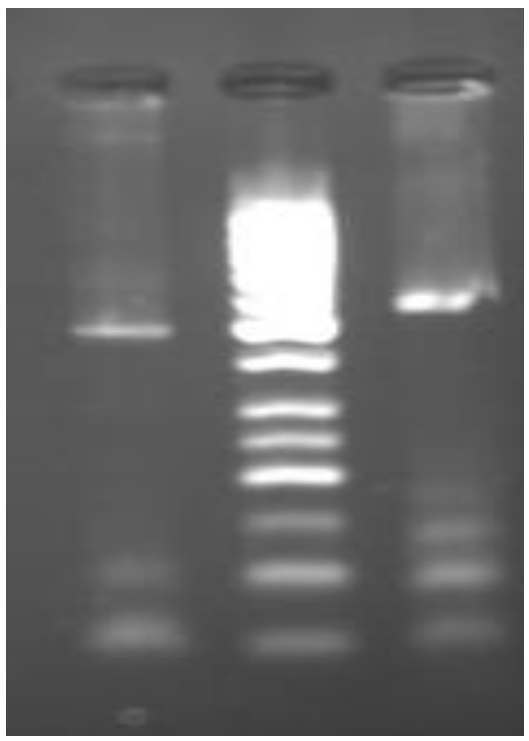

Figure 2. Real-time inverse PCR melting curves analysis of most of samples: 7 cases with INV22 mutations $\left(\mathrm{Tm}=83^{\circ} \mathrm{C}\right)$ and 4 cases without ones $\left(\mathrm{Tm}=85^{\circ} \mathrm{C}\right)$.

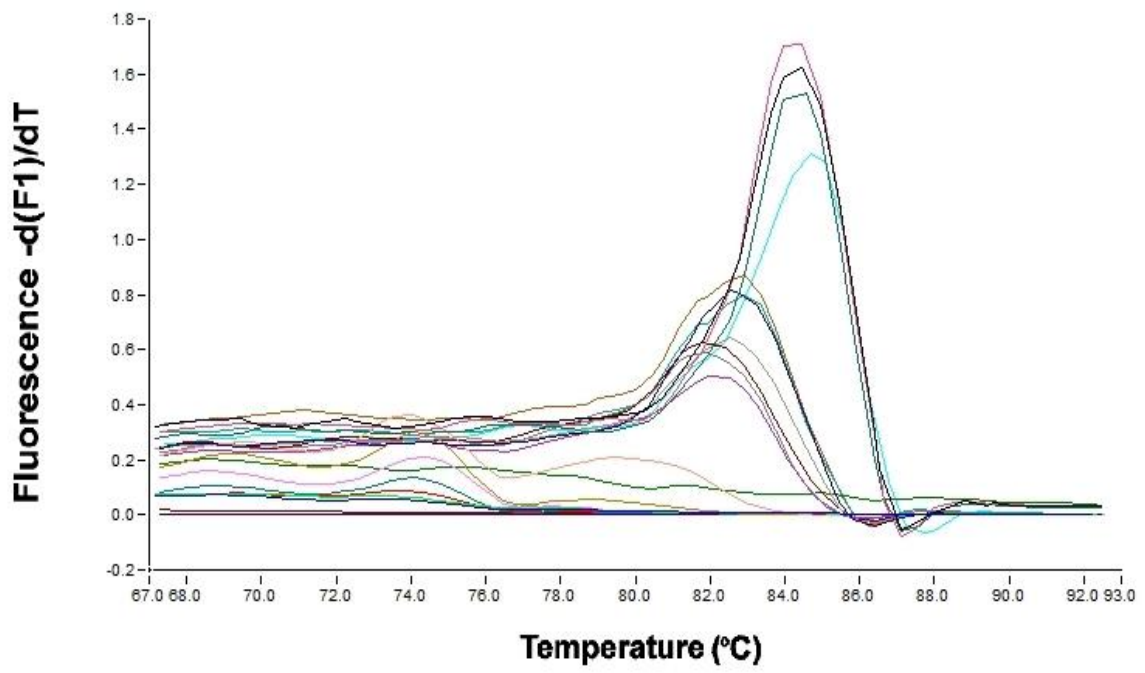

